\title{
Confined dipole and exchange spin waves in a bulk chiral magnet with Dzyaloshinskii-Moriya interaction
}

\author{
Ping Che $\odot,{ }^{1}$ Ioannis Stasinopoulos $\odot,{ }^{2}$ Andrea Mucchietto $\odot,{ }^{1}$ Jianing Li, ${ }^{1}$ Helmuth Berger, ${ }^{3}$ Andreas Bauer $\odot,{ }^{4,5}$ \\ Christian Pfleiderer, ${ }^{4,5,6}$ and Dirk Grundler ${ }^{1,7, *}$ \\ ${ }^{1}$ Laboratory of Nanoscale Magnetic Materials and Magnonics, Institute of Materials (IMX), École Polytechnique Fédérale de Lausanne \\ (EPFL), 1015 Lausanne, Switzerland \\ ${ }^{2}$ Physik Department E10, Technische Universität München, 85748 Garching, Germany \\ ${ }^{3}$ Institut de Physique de la Matière Complexe, École Polytechnique Fédérale de Lausanne (EPFL), 1015 Lausanne, Switzerland \\ ${ }^{4}$ Physik Department E51, Technische Universität München, 85748 Garching, Germany \\ ${ }^{5}$ Zentrum für QuantumEngineering (ZQE), Technische Universität München, D-85748 Garching, Germany \\ ${ }^{6}$ Munich Center for Quantum Science and Technology (MCQST), Technische Universität München, D-85748 Garching, Germany \\ ${ }^{7}$ Institute of Electrical and Micro Engineering, École Polytechnique Fédérale de Lausanne (EPFL), 1015 Lausanne, Switzerland
}

(Received 14 April 2021; revised 3 June 2021; accepted 3 June 2021; published 30 July 2021)

\begin{abstract}
The Dzyaloshinskii-Moriya interaction (DMI) has an impact on excited spin waves in the chiral magnet $\mathrm{Cu}_{2} \mathrm{OSeO}_{3}$ by means of introducing asymmetry in their dispersion relations. The confined eigenmodes of a chiral magnet are hence no longer the conventional standing spin waves. Here we report a combined experimental and micromagnetic modeling study by broadband microwave spectroscopy, and we observe confined spin waves up to eleventh order in bulk $\mathrm{Cu}_{2} \mathrm{OSeO}_{3}$ in the field-polarized state. In micromagnetic simulations we find similarly rich spectra. They indicate the simultaneous excitation of both dipole- and exchange-dominated spin waves with wavelengths down to $(47.2 \pm 0.05) \mathrm{nm}$ attributed to the exchange interaction modulation. Our results suggest the DMI to be effective in creating exchange spin waves in a bulk sample without the challenging nanofabrication and thereby in exploring their scattering with noncollinear spin textures.
\end{abstract}

DOI: 10.1103/PhysRevResearch.3.033104

\section{INTRODUCTION}

Magnon band structures are nontrivial in chiral magnets because of bulk Dzyaloshinskii-Moriya interaction (DMI) [1-6]. As a consequence of the asymmetric exchange interaction, bulk DMI introduces nonreciprocity for the spin waves [5,7-13]. Therefore chiral magnets can serve as nonreciprocal microwave devices [9]. In order to make use of the nontrivial magnonic properties, finite wave vector $k$ excitations in chiral magnets are strongly demanded. The spin dynamics in bulk chiral magnets with lateral dimensions up to millimeters have been investigated experimentally [14-21] but mostly focusing on a wave vector of $k=0$. To explore the spin wave excitation with finite $k$, focused ion beam patterning was utilized to shape the chiral magnets into lamella and to flip them onto microstructured coplanar waveguides (CPWs) [9,13]. Still, exchange-dominated spin waves in the gigahertz $(\mathrm{GHz})$ frequency regime are largely unexplored.

\footnotetext{
*dirk.grundler@epfl.ch

Published by the American Physical Society under the terms of the Creative Commons Attribution 4.0 International license. Further distribution of this work must maintain attribution to the author(s) and the published article's title, journal citation, and DOI.
}

In bulk magnets without the DMI, spin waves exhibit symmetric dispersion relations for $+k$ and $-k$. Standing spin waves form with fixed nodes and antinodes. Intensities of corresponding spin wave resonances vary systematically with the order number (number of nodal lines) $n$ (Supplemental Material, Fig. S1 [22]) [23-31]. In the chiral magnets with bulk DMI, the backward volume magnon dispersion relation is asymmetric [Fig. 1(a)] so that the spin waves propagating along opposite directions at the same frequency own different $k$. The discrepancy in $k$ does not allow for the conventional standing spin waves. Numerical methods showed involved spin waves' phase profiles in nanoscale magnets with the DMI [32-34]. In thin films of the chiral magnet $\mathrm{FeGe}$, an oscillating factor, $\exp (-i Q z)$, was introduced when the ferromagnetic resonance (FMR) at $k=0$ and the perpendicular standing spin waves with finite $k$ were interpreted, where $Q$ is the pitch vector in a chiral magnet [35]. Standing spin waves were assumed in bulk $\mathrm{Cu}_{2} \mathrm{OSeO}_{3}[18,19]$ but their characteristics remained unexplored. $\mathrm{Cu}_{2} \mathrm{OSeO}_{3}$ is among the best candidate materials for spin dynamics exploration in chiral magnets because it has a rich magnetic phase diagram [36-39] and a low damping. At small magnetic fields, noncollinear spin structures in the helical, conical, and skyrmion phases appear which exhibit anisotropic magnon band structures consisting of several allowed minibands and forbidden frequency gaps which vary significantly depending on the direction of the spin-wave wave vector $\mathbf{k}[4,13]$. The damping parameter $\alpha$ of $\mathrm{Cu}_{2} \mathrm{OSeO}_{3}$ was reported to be the lowest in the so far 
(a)

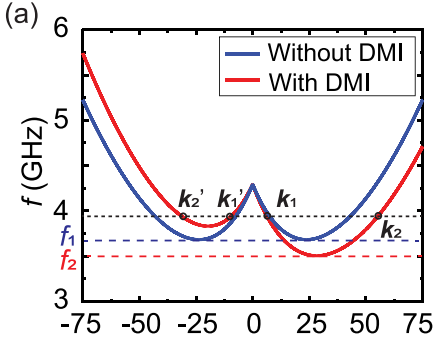

(b)

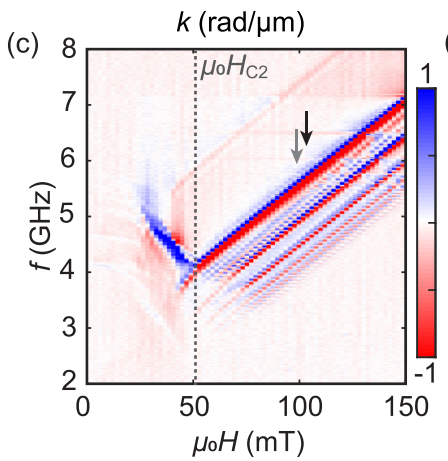

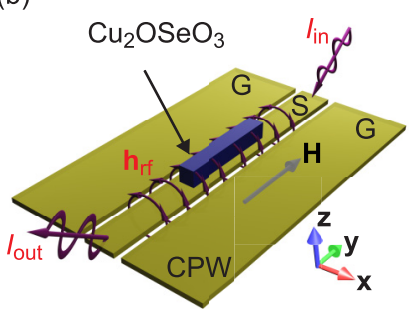

(d)

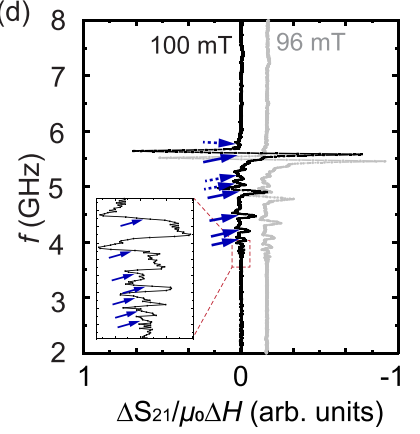

FIG. 1. (a) Sketch of the dispersion relation of $\mathbf{k} \| \mathbf{H}$ mode with and without the DMI. (b) Schematic diagram of bulk $\mathrm{Cu}_{2} \mathrm{OSeO}_{3}$ sample placed on a CPW (yellow colored). (c) Color-coded maps of broadband spin wave spectra taken as a function of the applied field $\mu_{0} H$ along $\mathbf{y}$ at $5 \mathrm{~K}$. The color scale bar represents $\Delta S_{12} / \mu_{0} \Delta H\left(\times 10^{-3} \mathrm{~T}^{-1}\right)$. Black and gray arrows indicate where the line plots in panel (d) were taken. (d) Line plots of spectra $\Delta S_{12} / \mu_{0} \Delta H$ at $5 \mathrm{~K}$ and $\mu_{0} H=100 \mathrm{mT}$ (black line) and $96 \mathrm{mT}$ (gray line) indicated by arrows in panel (c). Solid blue (and light blue) arrows mark the frequencies which we attribute to discrete $\mathbf{k} \| \mathbf{H}$ modes and dashed blue (and light blue) arrows mark the frequencies that we categorize in terms of discrete $\mathbf{k} \perp \mathbf{H}$ modes.

discovered skyrmion hosting materials. In particular in the field-polarized phase at $T=5 \mathrm{~K}$, the small value of $\alpha=1 \times$ $10^{-4}$ has been found [40] which allows for long decay lengths and spin-wave confinement in all three spatial directions of a macroscopic sample.

Here, we report on confined spin waves observed in the bulk chiral magnet $\mathrm{Cu}_{2} \mathrm{OSeO}_{3}$ probed by broadband microwave spectroscopy and micromagnetic simulation. To explore the combined effect of the DMI and sample boundaries on the formation of confined spin wave states we analyzed in detail the spectra obtained in the fieldpolarized state exhibiting low damping. At the same time, we avoided additional effects such as magnetic domain formation and noncollinear spin structures with highly anisotropic band structures $[4,18,41]$. We excited spin waves by a dynamic field across the whole sample [Fig. 1(b)] and numerous resonance peaks with systematically varying intensities appeared in the field-polarized phase for $\mu_{0} H>50 \mathrm{mT}$ in Fig. 1(c). We attributed the series of peaks to confined magnetostatic waves with wave vectors $\mathbf{k} \| \mathbf{H}$ and $\mathbf{k} \perp \mathbf{H}$. We performed micromagnetic simulations on a micron-sized sample exhibiting uniform magnetization and the same form factor, i.e., the same shape anisotropy (demagnetization effect). When considering a nonzero DMI, we observed both the odd and even order numbers $n$ of confined volume modes at low frequency

consistent with the experimental observation. The simulation reveals the short-waved magnons down to a wavelength of about $40 \mathrm{~nm}$ coexisting at frequencies of the discretized volume modes. These modes depended characteristically on the DMI strength. The origin of the short-waved magnons in the exchange regime is attributed to the interplay of symmetric and asymmetric exchange interactions. They appear for both uniform and nonuniform excitation scenarios. Our results suggest the DMI to be a strong tool to excite exchange-dominated spin waves without challenging nanofabrication.

\section{EXPERIMENTAL TECHNIQUE AND SIMULATIONS}

The broadband microwave measurements were conducted on a bar-shaped $\mathrm{Cu}_{2} \mathrm{OSeO}_{3}$ sample. The volume of the sample was $1 \mathrm{~mm} \times 0.29 \mathrm{~mm} \times 0.29 \mathrm{~mm}$ and all three surfaces were perpendicular to the easy axes [100], [010], and [001]. It was placed on a commercial CPW, which contained a 1-mm-wide signal line and two ground lines as sketched in Fig. 1(b). A radio-frequency current $I_{\mathrm{rf}}$ was injected into the CPW by a vector network analyzer (VNA) and induced a dynamic magnetic field $\mathbf{h}_{\mathrm{rf}}$ (purple circular arrows). The $\mathrm{Cu}_{2} \mathrm{OSeO}_{3}$ sample was positioned at the center of the CPW in a way that the longer axis of the sample was parallel to the signal line. Because the width of the signal line was $1 \mathrm{~mm}$ and more than three times wider compared to the sample width $\Delta x$ of $0.29 \mathrm{~mm}$, the in-plane component of $\mathbf{h}_{\mathrm{rf}}$ was assumed to be uniform with respect to $\mathbf{x}$ and $\mathbf{y}$ (it varied as a function of $\mathbf{z}$ ) [20]. Measurements were conducted at two cryogenic temperatures $T: 5$ and $20 \mathrm{~K}$ (Supplemental Material [22]). Magnetic fields were applied by a superconducting magnet along the $\mathbf{y}$ direction. A residual magnetic field of $36 \mathrm{mT}$ along $\mathbf{z}$ was present due to remanence of the setup when the sample was cooled down. The reported field values $\mu_{0} H$ describe the additional field applied via a current in the superconducting coil. The spectra discussed in this paper were taken at $\mu_{0} H>$ $96 \mathrm{mT}$; i.e., the applied field dominated the dynamic response. At temperature $T=5 \mathrm{~K}, \mu_{0} H=150 \mathrm{mT}$ was first applied along $+\mathbf{y}$ to saturate the $\mathrm{Cu}_{2} \mathrm{OSeO}_{3}$ and then reduced in a stepwise manner. The same saturation process was conducted when we changed the applied field direction to $\mathbf{H} \| \mathbf{x}$ and H $\| \mathbf{z}$ for different measurements. The sample was heated to $20 \mathrm{~K}$ to perform the same series of measurements with the different field directions at different $T$. Again we focused on the spin dynamics in the field-polarized phase to avoid domains and noncollinear spin structures.

In order to explore the role of the DMI for confined spin waves, we conducted spin dynamics simulations using the micromagnetic software MUMAX3 [42] assuming $T=0$. A bar-shaped sample with $0.128 \times 2.048 \times 0.128 \mu \mathrm{m}^{3}$ was considered. The cell sizes were $4 \mathrm{~nm}$ along $\mathbf{y}$ and $8 \mathrm{~nm}$ along $\mathbf{x}$ and $\mathbf{z}$ to resolve quantized spin waves. Here we used $1 \leqslant i x \leqslant 16,1 \leqslant i y \leqslant 512$ and $1 \leqslant i z \leqslant 16$ to identify the cell number along the $\mathbf{x}, \mathbf{y}$, and $\mathbf{z}$ directions. Parameters were as follows: exchange stiffness $A_{s}=7 \times 10^{-13} \mathrm{~J} / \mathrm{m}$, DMI constant $D=7.4 \times 10^{-5} \mathrm{~J} / \mathrm{m}$, saturation magnetization $M_{\text {sat }}=$ $1.03 \times 10^{5} \mathrm{~A} / \mathrm{m}$, first-order cubic anisotropy constant $K_{\mathrm{C} 1}=$ $6 \times 10^{2} \mathrm{~J} / \mathrm{m}^{3}$, and Gilbert damping constant $\alpha=5 \times 10^{-4}$ modeled $\mathrm{Cu}_{2} \mathrm{OSeO}_{3}$; they were taken from Refs. [40,43,44]. 
In additional simulations, we varied the parameter $D$ between 0 and $10\left(\times 10^{-5} \mathrm{~J} / \mathrm{m}^{2}\right)$. The static magnetic field $\mu_{0} H$ was varied from 160 to $80 \mathrm{mT}$ along $\mathbf{y}$ with a $0.5^{\circ}$ tilting angle with respect to $\mathbf{x}$ to avoid numerical errors. The micromagnetic simulations do not consider the residual field in the experiment. The dynamic magnetic field $\mu_{0} h$ in the format of a sinc function for broadband excitation was applied along $\mathbf{x}$ and $0.5^{\circ}$ tilted to $\mathbf{z}$.

\section{DISPERSION RELATION MODIFIED BY BULK DZYALOSHINSKII-MORIYA INTERACTION}

Before we present results, it is instructive to look into the dispersion relations modified by bulk DMI compared with the conventional case in Fig. 1(a) $[8,45]$. In the ferrimagnetic state, the effective field term generated by bulk DMI enters the dispersion relation of only the spin wave mode with k $\| \mathbf{H}$ and has no influence on the spin wave mode with $\mathbf{k} \perp \mathbf{H}$. With bulk DMI $D>0$, the $\mathbf{k} \| \mathbf{H}$ mode dispersion relation is asymmetric with respect to $k=0 \mathrm{rad} / \mu \mathrm{m}$ : the branch with positive $k$ has a frequency lower than that of the system with no DMI and the branch with negative $k$ has a higher frequency. In Fig. 1(a) the asymmetry due to the DMI is exaggerated to make the difference in dispersion relations visible. When the bulk DMI is absent, the wave vectors at a given frequency have the same modulus of different sign, so a standing spin wave with vector $\left|k_{0}\right|=\left(\left|k_{+}\right|+\left|k_{-}\right|\right) / 2$ can form with fixed nodes and antinodes in a sample with finite length (width) $L$. In a one-dimensional case, $\left|k_{0}\right|$ is specified as $\left|k_{0}\right|=n \pi / L$, where $n$ is the number of nodes $0,1, \ldots$ [31]. If no specific surface asymmetry plays a role, broadband spin wave spectroscopy based on a wide CPW can barely detect the odd modes because the antinodes with $\pi$ phase shift induce counteracting voltage signals in the signal line of the CPWs which cancel each other. When bulk DMI is present, the dispersion relation is no longer symmetric, so that at each frequency $\left|k_{1}\right| \neq\left|k_{1}^{\prime}\right|$ and $\left|k_{2}\right| \neq\left|k_{2}^{\prime}\right|$. Traveling waves with fixed nodes are expected to form but spin wave amplitudes have "a pronounced time-dependent asymmetry in the mode profile" [33]. In this scenario, the voltage signals induced by the odd modes do not cancel and one expects an induced net voltage in the CPW. When analyzing our experimental data, we discuss $\left|k_{0}\right|=\left(\left|k_{1}\right|+\left|k_{1}^{\prime}\right| / 2\right)$ and not the individual values $\left|k_{1}\right|$ and $\left|k_{1}^{\prime}\right|$. This is because the excitation takes place at a fixed frequency and multiple $k\left(\left|k_{1}\right|\right.$ and $\left.\left|k_{1}^{\prime}\right|\right)$ are excited at the same time. In the micromagnetic simulations (methods), we analyze the integrated Fourier transform of spin wave amplitudes consistent with the signal detected by a CPW. With the help of the simulated phase profiles, we extract $\left|k_{0}\right|$ by a fitting procedure.

\section{BROADBAND SPIN WAVE SPECTROSCOPY DATA}

Field-dependent spectra taken at $T=5 \mathrm{~K}$ are shown in Fig. 1(c) for $\mathbf{H} \| \mathbf{x}$. A change in the slope $d f / d H$ of resonance frequencies $f$ was seen at $\mu_{0} H_{\mathrm{C} 2}=52 \mathrm{mT}$, marked by a black dashed line. For $\mu_{0} H>\mu_{0} H_{\mathrm{C} 2}$, resonance frequencies $f$ increased almost linearly with $H$, indicating the field-polarized phase. For $\mu_{0} H<\mu_{0} H_{\mathrm{C} 2}$, the slope $d f / d H$ indicated an unsaturated state, such as the conical phase in $\mathrm{Cu}_{2} \mathrm{OSeO}_{3}$ [15]. In the following we focus on the numerous resonances detected for $\mu_{0} H>\mu_{0} H_{\mathrm{C} 2}$. Line spectra at fields $\mu_{0} H=100 \mathrm{mT}$ and $\mu_{0} H=96 \mathrm{mT}$ are shown in Fig. 1(d). Fifteen peaks were observed and are marked by arrows. A color-coded map of spin wave spectra at $T=20 \mathrm{~K}$ and corresponding line spectra at $\mu_{0} H=112 \mathrm{mT}$ and $\mu_{0} H=108 \mathrm{mT}$ are shown in the Supplemental Material, Fig. S2 [22]. At $20 \mathrm{~K}$, five peaks (marked by arrows) were clearly visible above $\mu_{0} H_{\mathrm{C} 2}$. The small number of resonance is attributed to an increased damping, consistent with earlier reports on temperature-dependent damping $[13,40]$.

We have categorized the observed resonances depending on their systematic peak-to-peak intensity variation. Solid arrows mark $\mathbf{k} \| \mathbf{H}$ modes with different wave vectors $k_{y}$. The sequence of peaks was ranked with order numbers 1 , $2, \ldots$, with intensity $I$ varying from high to low. The intensities $I$ of resonances marked by solid arrows are summarized in Fig. 2(a) in a double-logarithmic manner. The linear variation suggests that $I$ is inversely proportional to the order number, which is a sign of confined spin waves with different numbers of nodes [30]. Considering $\mathbf{k} \| \mathbf{H}$ modes to be confined along $\mathbf{y}$ we attribute wave vectors to the discrete modes of Fig. 1(d) according to $k_{y}=\left(\pi n_{y}\right) / \Delta y$, where $n_{y}=0,1, \ldots$ and $\Delta y=L=1 \mathrm{~mm}$ is the length of the bar-shaped sample along the magnetic field direction. Figure 2(b) now shows the resonance frequencies identified in Fig. 1(d) as a function of estimated values $k_{y}$ (blue squares). The frequencies follow $f(k)$ expected for $\mathbf{k} \| \mathbf{H}$ modes. The group velocities $v_{\mathrm{g}}$ calculated from panel (b) according to $v_{\mathrm{g}}=2 \pi \Delta f / \Delta k_{y}$ are shown in Fig. 2(c). We find a value $v_{\mathrm{g}}$ of $-1300 \mathrm{~km} / \mathrm{s}$ near $k=0$. This value reflects a backward volume magnetostatic wave in the long-wavelength limit in the dipolar regime of a relatively thick ferrimagnet.

Dashed arrows in Fig. 1(d) mark additional discretized $\mathbf{k} \perp \mathbf{H}$ modes formed on top of $\mathbf{k} \| \mathbf{H}$ modes. The combined wave vector of such excitations reads $k^{2}=k_{y}{ }^{2}+k_{\perp}{ }^{2}$, where $k_{\perp}$ represents the wave vector in a direction transverse to the magnetic field vector [46]. The peaks marked by dashed arrows follow the sequence of conventional standing spin waves because the bulk DMI does not modify the dispersion relation of the spin waves when $\mathbf{k} \perp \mathbf{H}$ and those modes exhibit a positive group velocity. Hence higher-order confined modes exhibit higher resonance frequencies. $\mathbf{k} \perp \mathbf{H}$ modes confined along $\mathbf{z}$ and $\mathbf{x}$ are degenerate because the dimensions of the considered bulk $\mathrm{Cu}_{2} \mathrm{OSeO}_{3}$ sample amount to $\Delta z=$ $\Delta x=0.29 \mathrm{~mm}$.

The colored-coded maps of broadband spin wave spectra for other field directions are plotted in Fig. S3 of the Supplemental Material [22]. When the field was applied along $\mathbf{z}$, only one further quantized $\mathbf{k} \| \mathbf{H}$ mode was resolved at lower frequency because the wave vector determined by the dimension $\Delta z=0.29 \mathrm{~mm}$ is much larger and may approach the bottom of the $\mathbf{k} \| \mathbf{H}$ magnetostatic wave band. The dispersion relations and group velocities are consistent for the configuration of $\mathbf{H} \| \mathbf{z}$ [Figs. 2(b) and 2(c)]. When the field was applied along $\mathbf{x}$, a confined $\mathbf{k} \| \mathbf{H}$ mode was not resolved, most likely due to a vanishing torque because of $\mathbf{h}_{\mathrm{rf}} \|$ M. Still the FMR and edge modes were observed. The intensities were low. 

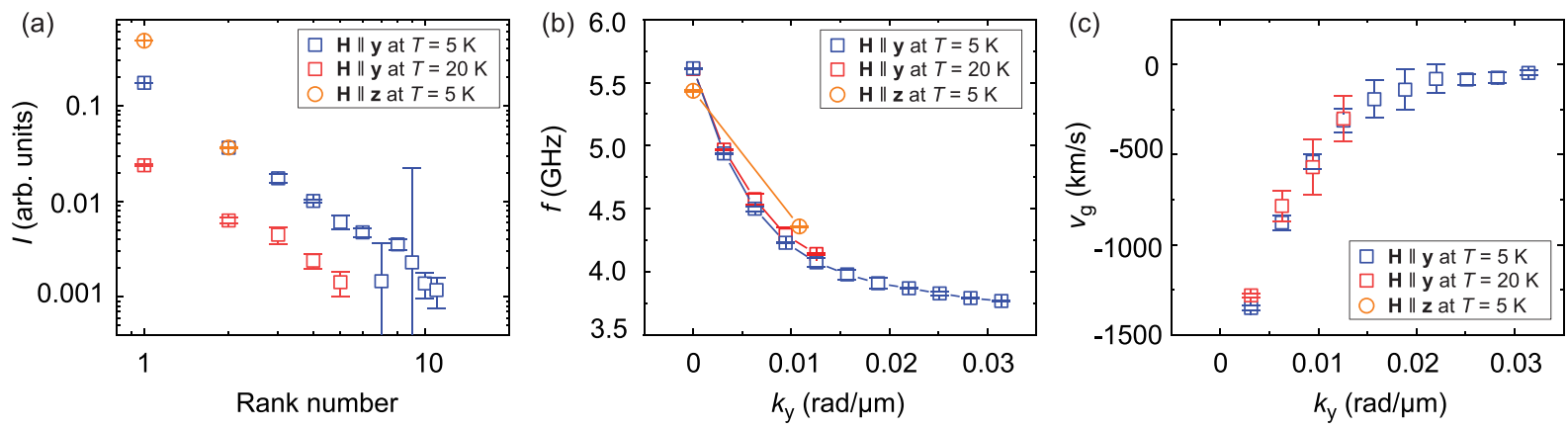

FIG. 2. (a) Peak-to-peak intensity of resonances in a double-logarithmic plot corresponding to confined $\mathbf{k} \| \mathbf{H}$ mode sequences. Error bars represent the noise level in the spectra and the deviation in reading out the peaks. (b) Dispersion relation of spin waves extracted experimentally. Error bars reflect the frequency resolution of the VNA and the deviation in reading out the peaks. (c) Group velocities calculated as $2 \pi \Delta f / \Delta k_{y}$ from panel (b). Error bars originate from the frequency uncertainty in panel (b). In panels (a), (b), and (c), blue squares were extracted from the solid blue arrows in Fig. 1(d) of $\mathbf{H} \| \mathbf{y}$; red squares were extracted from data taken at $\mu_{0} H=112 \mathrm{mT}$ at $T=20 \mathrm{~K}$ (Supplemental Material, Fig. S2 [22]); yellow circles were extracted from data taken at $\mu_{0} H=150 \mathrm{mT}$ at $T=5 \mathrm{~K}$ when $\mathbf{H} \| \mathbf{z}$ (Supplemental Material, Fig. S3 [22]). Spin-wave dispersion relations evaluated from three different experiments are consistent; see panel (b).

\section{SIMULATED MODES AND DISCUSSION}

The integrated Fourier transform (FFT) amplitude of the magnetization component $\left|m_{z}\right|$ of all cells is plotted in Figs. 3 and $4(\mathrm{a})$. In both data sets we find one mode of largest intensity. On the low-frequency side of this most prominent peak, multiple resonance peaks are seen. This is consistent with the observation in the experiment [Fig. 1(c)]. It is noted that when the periodic boundary condition (PBC) was applied along $\mathbf{y}$, there were no multiple peaks at lower frequency in the simulation. Therefore, the resonance peaks at lower frequency reflected discrete spin waves confined by the sample boundaries in the $y$ direction.

The bulk DMI constant was varied from 0 to 10 $\left(\times 10^{-5} \mathrm{~J} / \mathrm{m}^{2}\right)$ in Fig. 3. Here the applied field was fixed at $160 \mathrm{mT}$. Multiple peaks with decreasing amplitudes towards lower frequency are present for all the spectra, substantiating the formation of discrete modes due to spin wave confinement between sample boundaries for all the different values of $D$. When $D=0$, confined spin waves reside between 4.4 and $7.3 \mathrm{GHz}$ and the highest intensity peak appears at

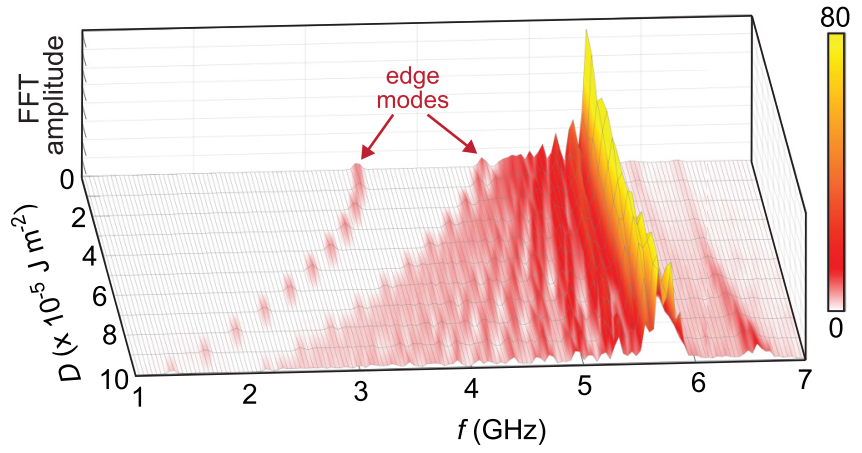

FIG. 3. Spin wave spectra for different DMI constants. The color bar represents the integrated Fourier transform (FFT) amplitude of the magnetization component $m_{z}$.
6.5 GHz. The discrete $\mathbf{k} \| \mathbf{H}$ mode resonances reflect numbers $n_{y}=1,3,5, \ldots$ On the one hand, when $D$ increases up to $10 \times 10^{-5} \mathrm{~J} / \mathrm{m}^{2}$, the peak with the highest intensity moves to a higher frequency of $6.8 \mathrm{GHz}$ and more $\mathbf{k} \| \mathbf{H}$ mode resonance peaks appear down to $2.3 \mathrm{GHz}$ (instead of $4.4 \mathrm{GHz}$ ). As shown in Fig. 1(a), when bulk DMI is present, the dispersion relation $f(k)$ of $\mathbf{k} \| \mathbf{H}$ magnetostatic waves is asymmetric. This asymmetry increases with $D$. For $D \neq 0$, the lowest frequency $f_{2}$ possible to be excited is lower than $f_{1}$, which denotes the bottom of the symmetric $\mathbf{k} \| \mathbf{H}$ mode band in the case of $D=0$. The lower frequency $f_{2}$ explains the larger frequency regime in which discrete $\mathbf{k} \| \mathbf{H}$ modes occur for increasing $D$. On the other hand, the asymmetry results in the occurrence of both the even and odd-numbered modes $n_{y}$ in the integrated FFT amplitudes of our simulation. Thus all the confined $\mathbf{k} \| \mathbf{H}$ modes with $n_{y}=1,2,3, \ldots$ appear and the total number of resonance peaks increases with $D$.

For $f=6 \mathrm{GHz}$ and $D=7.4 \times 10^{-5} \mathrm{~J} / \mathrm{m}^{2}$ we plot the simulated spin-precessional amplitudes $A$ and phases $\Phi$ inside the chiral magnet (for layer $i z=6$ ) in Fig. 4(b). In both the $A$ and $\Phi$ maps, a confined long-waved spin wave (marked by a dashed red arrow) and a short-waved spin wave (marked by solid red arrows) are resolved. The phase line plots for $i z=6$ and $i x=6$ of all the resonances at $160 \mathrm{mT}$ are plotted in Fig. S4 of the Supplemental Material [22]. All of them display the coexistence of two waves with different wavelengths. We label them as long-waved spin waves (LWSWs) and short-waved spin waves (SWSWs) in the following.

The simulated resonance frequencies of both LWSWs and SWSWs are summarized in Fig. 4(c). The open symbols representing the LWSWs follow the dispersion relation of the dipolar $\mathbf{k} \quad \| \mathbf{H}$ magnetostatic waves with a negative group velocity consistent with the experimental results shown in Fig. 2(b). It is notable that the wave vectors of LWSWs do not match perfectly with the calculation in experimentally extracted data as $k_{y}=\left(\pi n_{y}\right) / \Delta y\left(n_{y}=0,1, \ldots\right.$ and $\Delta y$ is the sample length). We interpret the reason to be the inhomogeneity of the internal field in the simulated sample 


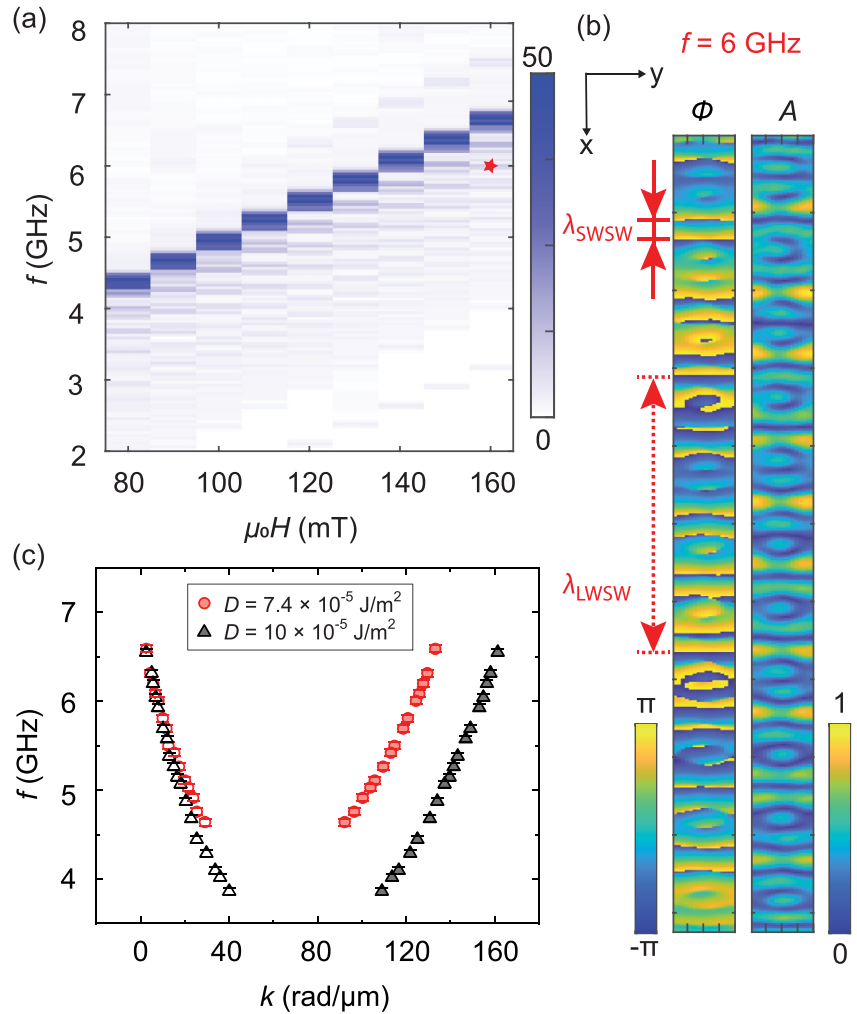

FIG. 4. (a) Color-coded maps of spin wave spectra from micromagnetic simulations in layer 6 in the $\mathbf{z}$ direction. The color scale bar represents the amplitude of Fourier transform of $m_{z}$. (b) Color-coded maps of amplitude $A$ and phase $\Phi$ in the $x-y$ plane of the $z$ layer $i z=6$, with $D=7.4 \times 10^{-5} \mathrm{~J} / \mathrm{m}^{2}$ at the field value indicated in panel (a) by the red star. The color scales of amplitude maps are normalized so that blue represents 0 and yellow represents 1 . In the phase maps, blue and yellow represent $-\pi$ and $\pi$, respectively. The wavelengths of the SWSW and LWSW are marked. (c) Dispersion relation of resonance frequencies extracted from the micromagnetic simulations. Red squares are taken from $\mu_{0} H=160 \mathrm{mT}$ with $D=$ $7.4 \times 10^{-5} \mathrm{~J} / \mathrm{m}^{2}$. Black triangles are taken from $\mu_{0} H=160 \mathrm{mT}$ with $D=10 \times 10^{-5} \mathrm{~J} / \mathrm{m}^{2}$. Open symbols are LWSWs and correspond to the dashed red arrow in panel (b). Solid symbols are SWSWs and correspond to the solid red arrows in panel (b).

near the edges which modifies the wave format. It is not counted in the calculation of wave vectors in Fig. 2 because the sample dimension along $\mathbf{y}$ in experiment is as large as $y=1 \mathrm{~mm}$ and thus the influence of the inhomogeneous region is negligible. The solid symbols representing SWSWs show the characteristics of exchange-dominated spin waves with a positive group velocity. In the case of $D=$ $7.4 \times 10^{-5} \mathrm{~J} / \mathrm{m}^{2}$ modeling $\mathrm{Cu}_{2} \mathrm{OSeO}_{3}$, the largest resolved wave vector amounts to $k=133 \mathrm{rad} / \mu \mathrm{m}$ corresponding to a wavelength of $\lambda=(47.2 \pm 0.05) \mathrm{nm}$. When we increase the DMI constant to $D=10 \times 10^{-5} \mathrm{~J} / \mathrm{m}^{2}$, the maximum wave vector of the exchange-dominated SWSWs increases to $k=162 \mathrm{rad} / \mu \mathrm{m}$, corresponding to $\lambda=(38.9 \pm 0.04) \mathrm{nm}$. However, the wavelengths of the LWSWs remain similar to the smaller DMI value. The small deviation is because of the enhancement of the asymmetry of the $\mathbf{k} \| \mathbf{H}$ mode dispersion relation. The sign of the DMI does not play a role for the extracted absolute wave vector values but it changes the sign of the phase velocity of both LWSWs and SWSWs (see the Supplemental Material, Fig. S6 [22]). We attribute the coexistence of multiple wave vector excitations at each frequency in the simulations to the exchange interactions. The asymmetric DMI, together with the symmetric exchange interaction, creates a periodic modulation of exchange interactions for spins in chiral magnets and the period is controlled by the strength of the DMI constant $D$ and the exchange stiffness $A$. When the confined $\mathbf{k} \| \mathbf{H}$ modes with $k_{1}$ are excited because of the sample boundaries, the modulation of exchange interactions provides the source of $k_{2}$ excitation at the same frequency. Even in the case of a small DMI which might not be sufficient to induce skyrmions, we observe modifications of spectra (Fig. 3). They show that bulk DMI apart from the creation of the noncollinear phases has a sizable effect on the spin wave excitation and the nature of standing spin waves. Our results are important when exploring spin-wave confinement effects in noncollinear spin structures which exhibit anisotropic minibands and forbidden frequency gaps.

\section{CONCLUSION}

In summary, we reported confined magnetostatic waves formed by the sample boundaries in a bar-shaped bulk $\mathrm{Cu}_{2} \mathrm{OSeO}_{3}$ sample explored by both broadband spin wave spectroscopy and micromagnetic simulations in the fieldpolarized state. In the simulations, ultrashort spin waves down to $(47.2 \pm 0.05) \mathrm{nm}$ were predicted beyond the experimentally observed dipolar spin wave modes with $\mathbf{k} \|$ H. They were attributed to the DMI-induced asymmetry of the dispersion relation and the periodic modulation of exchange interactions in chiral magnets. It has been proved that the wavelength and the phase velocity strongly depend on the DMI strength. By increasing the DMI strength, shorter wavelengths have been achieved. Our findings provide an alternative way of exchange-dominated spin wave excitation without the need of nanofabrication.

The data that support the findings of this study are openly available in Zendo at [47].

\section{ACKNOWLEDGMENTS}

The authors thank Professor Markus Garst from Karlsruhe Institute of Technology for the discussions and comments on the manuscript. We acknowledge financial support from the Swiss National Science Foundation (SNSF) via Grant No. 171003 Sinergia project Nanoskyrmionics, the Deutsche Forschungsgemeinschaft (DFG, German Research Foundation) under Grant No. TRR80 (From Electronic Correlations to Functionality, Project No. 107745057, and Projects No. E1 and No. F7), SPP2137 (Skyrmionics, Project No. 403191981, Grant No. PF393/19), and the excellence cluster MCQST under Germany's Excellence Strategy EXC-2111 (Project No. 390814868). Financial support by the European Research Council (ERC) through Advanced Grants No. 291079 (TOPFIT) and No. 788031 (ExQuiSid) is gratefully acknowledged. 
[1] M. Kugler, G. Brandl, J. Waizner, M. Janoschek, R. Georgii, A. Bauer, K. Seemann, A. Rosch, C. Pfleiderer, P. Böni, and M. Garst, Band Structure of Helimagnons in MnSi Resolved by Inelastic Neutron Scattering, Phys. Rev. Lett. 115, 097203 (2015).

[2] P. Y. Portnichenko, J. Romhányi, Y. A. Onykiienko, A. Henschel, M. Schmidt, A. S. Cameron, M. A. Surmach, J. A. Lim, J. T. Park, A. Schneidewind, D. L. Abernathy, H. Rosner, J. Van Den Brink, and D. S. Inosov, Magnon spectrum of the helimagnetic insulator $\mathrm{Cu}_{2} \mathrm{OSeO}_{3}$, Nat. Commun. 7, 10725 (2016).

[3] A. Roldán-Molina, A. S. Nunez, and J. Fernández-Rossier, Topological spin waves in the atomic-scale magnetic skyrmion crystal, New J. Phys. 18, 045015 (2016).

[4] M. Garst, J. Waizner, and D. Grundler, Collective spin excitations of helices and magnetic skyrmions: Review and perspectives of magnonics in non-centrosymmetric magnets, J. Phys. D: Appl. Phys. 50, 293002 (2017).

[5] T. Weber, J. Waizner, G. S. Tucker, R. Georgii, M. Kugler, A. Bauer, C. Pfleiderer, M. Garst, and P. Böni, Field dependence of nonreciprocal magnons in chiral MnSi, Phys. Rev. B 97, 224403 (2018).

[6] Y. Luo, G. G. Marcus, B. A. Trump, J. Kindervater, M. B. Stone, J. A. Rodriguez-Rivera, Y. Qiu, T. M. McQueen, O. Tchernyshyov, and C. Broholm, Low-energy magnons in the chiral ferrimagnet $\mathrm{Cu}_{2} \mathrm{OSeO}_{3}$ : A coarse-grained approach, Phys. Rev. B 101, 144411 (2020).

[7] M. Kataoka, Spin waves in systems with long period helical spindensity waves due to the antisymmetric and symmetric exchange interactions, J. Phys. Soc. Jpn. 56, 3635 (1987).

[8] D. Cortés-Ortuño, and P. Landeros, Influence of the Dzyaloshinskii-Moriya interaction on the spin-wave spectra of thin films, J. Phys.: Condens. Matter 25, 156001 (2013).

[9] S. Seki, Y. Okamura, K. Kondou, K. Shibata, M. Kubota, R. Takagi, F. Kagawa, M. Kawasaki, G. Tatara, Y. Otani, and Y. Tokura, Magnetochiral nonreciprocity of volume spin wave propagation in chiral-lattice ferromagnets, Phys. Rev. B 93, 235131 (2016).

[10] T. J. Sato, D. Okuyama, T. Hong, A. Kikkawa, Y. Taguchi, and Y. Tokura, Magnon dispersion shift in the induced ferromagnetic phase of noncentrosymmetric MnSi, Phys. Rev. B 94, 144420 (2016).

[11] T. Weber, J. Waizner, G. S. Tucker, L. Beddrich, M. Skoulatos, R. Georgii, A. Bauer, C. Pfleiderer, M. Garst, and P. Böni, Nonreciprocal magnons in non-centrosymmetric MnSi, AIP Adv. 8, 101328 (2018).

[12] T. Weber, J. Waizner, P. Steffens, A. Bauer, C. Pfleiderer, M. Garst, and P. Böni, Polarized inelastic neutron scattering of nonreciprocal spin waves in MnSi, Phys. Rev. B 100, 060404(R) (2019).

[13] S. Seki, M. Garst, J. Waizner, R. Takagi, N. D. Khanh, Y. Okamura, K. Kondou, F. Kagawa, Y. Otani, and Y. Tokura, Propagation dynamics of spin excitations along skyrmion strings, Nat. Commun. 11, 256 (2020).

[14] Y. Onose, Y. Okamura, S. Seki, S. Ishiwata, and Y. Tokura, Observation of Magnetic Excitations of Skyrmion Crystal in a Helimagnetic Insulator $\mathrm{Cu}_{2} \mathrm{OSeO}_{3}$, Phys. Rev. Lett. 109, 037603 (2012).

[15] T. Schwarze, J. Waizner, M. Garst, A. Bauer, I. Stasinopoulos, H. Berger, C. Pfleiderer, and D. Grundler, Universal heli- magnon and skyrmion excitations in metallic, semiconducting and insulating chiral magnets, Nat. Mater. 14, 478 (2015).

[16] Y. Okamura, F. Kagawa, M. Mochizuki, M. Kubota, S. Seki, S. Ishiwata, M. Kawasaki, Y. Onose, and Y. Tokura, Microwave magnetoelectric effect via skyrmion resonance modes in a helimagnetic multiferroic, Nat. Commun. 4, 2391 (2013).

[17] Y. Okamura, F. Kagawa, S. Seki, M. Kubota, M. Kawasaki, and Y. Tokura, Microwave Magnetochiral Dichroism in the Chiral-Lattice Magnet $\mathrm{Cu}_{2} \mathrm{OSeO}_{3}$, Phys. Rev. Lett. 114, 197202 (2015).

[18] M. Weiler, A. Aqeel, M. Mostovoy, A. Leonov, S. Geprägs, R. Gross, H. Huebl, T. T. M. Palstra, and S. T. B. Goennenwein, Helimagnon Resonances in an Intrinsic Chiral Magnonic Crystal, Phys. Rev. Lett. 119, 237204 (2017).

[19] A. Aqeel, J. Sahliger, T. Taniguchi, S. Maendl, D. Mettus, H. Berger, A. Bauer, M. Garst, C. Pleiderer, and C. H. Back, Microwave Spectroscopy of the Low-Temperature Skyrmion State in $\mathrm{Cu}_{2} \mathrm{OSeO}_{3}$, Phys. Rev. Lett. 126, 017202 (2021).

[20] I. Stasinopoulos, S. Weichselbaumer, A. Bauer, J. Waizner, H. Berger, M. Garst, C. Pfleiderer, and D. Grundler, Linearly polarized $\mathrm{GHz}$ magnetization dynamics of spin helix modes in the ferrimagnetic insulator $\mathrm{Cu}_{2} \mathrm{OSeO}_{3}$, Sci. Rep. 7, 7037 (2017).

[21] S. Pöllath, A. Aqeel, A. Bauer, C. Luo, H. Ryll, F. Radu, C. Pfleiderer, G. Woltersdorf, and C. H. Back, Ferromagnetic Resonance with Magnetic Phase Selectivity by Means of Resonant Elastic X-Ray Scattering on a Chiral Magnet, Phys. Rev. Lett. 123, 167201 (2019).

[22] See Supplemental Material at http://link.aps.org/supplemental/ 10.1103/PhysRevResearch.3.033104 for details regarding the confined spin waves in magnets without bulk DMI, broadband spin wave spectra at $T=20 \mathrm{~K}$ and variable field directions, the phase of quantized spin waves from micromagnetic simulation, the FFT of the phase and amplitude maps, and the influence of the DMI sign on the phase of quantized spin waves.

[23] J. Jorzick, S. O. Demokritov, C. Mathieu, B. Hillebrands, B. Bartenlian, C. Chappert, F. Rousseaux, and A. N. Slavin, Brillouin light scattering from quantized spin waves in micron-size magnetic wires, Phys. Rev. B 60, 15194 (1999).

[24] M. van Kampen, C. Jozsa, J. T. Kohlhepp, P. LeClair, L. Lagae, W. J. M. de Jonge, and B. Koopmans, All-Optical Probe of Coherent Spin Waves, Phys. Rev. Lett. 88, 227201 (2002).

[25] C. Bayer, J. Jorzick, B. Hillebrands, S. O. Demokritov, R. Kouba, R. Bozinoski, A. N. Slavin, K. Y. Guslienko, D. V. Berkov, N. L. Gorn, and M. P. Kostylev, Spin-wave excitations in finite rectangular elements of $\mathrm{Ni}_{80} \mathrm{Fe}_{20}$, Phys. Rev. B 72, 064427 (2005).

[26] V. E. Demidov, U.-H. Hansen, and S. O. Demokritov, SpinWave Eigenmodes of a Saturated Magnetic Square at Different Precession Angles, Phys. Rev. Lett. 98, 157203 (2007).

[27] V. E. Demidov, S. O. Demokritov, K. Rott, P. Krzysteczko, and G. Reiss, Mode interference and periodic self-focusing of spin waves in permalloy microstripes, Phys. Rev. B 77, 064406 (2008).

[28] M. Mruczkiewicz, M. Krawczyk, V. K. Sakharov, Y. V Khivintsev, Y. A. Filimonov, and S. A. Nikitov, Standing spin waves in magnonic crystals, J. Appl. Phys 113, 093908 (2013).

[29] D. A. Bozhko, H. Yu. Musiienko-Shmarova, V. S. Tiberkevich, A. N. Slavin, I. I. Syvorotka, B. Hillebrands, and A. A. Serga, Unconventional spin currents in magnetic films, Phys. Rev. Res. 2, 023324 (2020). 
[30] T. An, V. I. Vasyuchka, K. Uchida, A. V. Chumak, K. Yamaguchi, K. Harii, J. Ohe, M. B. Jungfleisch, Y. Kajiwara, H. Adachi, B. Hillebrands, S. Maekawa, and E. Saitoh, Unidirectional spin-wave heat conveyer. Nat. Mater. 12, 549 (2013).

[31] M. Grimsditch, A. Malozemoff, and A. Brunsch, Standing Spin Waves Observed by Brillouin Scattering in Amorphous Metallic $\mathrm{Fe}_{80} \mathrm{~B}_{20}$ Films, Phys. Rev. Lett. 43, 711 (1979).

[32] F. Garcia-Sanchez, P. Borys, A. Vansteenkiste, J.-V. Kim, and R. L. Stamps, Nonreciprocal spin-wave channeling along textures driven by the Dzyaloshinskii-Moriya interaction, Phys. Rev. B 89, 224408 (2014).

[33] B. W. Zingsem, M. Farle, R. L. Stamps, and R. E. Camley, Unusual nature of confined modes in a chiral system: Directional transport in standing waves, Phys. Rev. B 99, 214429 (2019).

[34] N. Josten, T. Feggeler, R. Meckenstock, D. Spoddig, M. Spasova, K. Chai, I. Radulov, Z. A. Li, O. Gutfleisch, M. Farle, and B. Zingsem, Dynamic unidirectional anisotropy in cubic FeGe with antisymmetric spin-spin-coupling, Sci. Rep. 10, 2861 (2020).

[35] S. L. Zhang, I. Stasinopoulos, T. Lancaster, F. Xiao, A. Bauer, F. Rucker, A. A. Baker, A. I. Figueroa, Z. Salman, F. L. Pratt, S. J. Blundell, T. Prokscha, A. Suter, J. Waizner, M. Garst, D. Grundler, G. van der Laan, C. Pfleiderer, and T. Hesjedal, Room-temperature helimagnetism in FeGe thin films, Sci. Rep. 7, 123 (2017).

[36] S. Seki, X. Z. Yu, S. Ishiwata, and Y. Tokura, Observation of skyrmions in a multiferroic material, Science 336, 198 (2012).

[37] Y. Okamura, Y. Yamasaki, D. Morikawa, T. Honda, V. Ukleev, H. Nakao, Y. Murakami, K. Shibata, F. Kagawa, S. Seki, T. Arima, and Y. Tokura, Emergence and magnetic-field variation of chiral-soliton lattice and skyrmion lattice in the strained helimagnet $\mathrm{Cu}_{2} \mathrm{OSeO}_{3}$, Phys. Rev. B 96, 174417 (2017).
[38] A. Chacon, L. Heinen, M. Halder, A. Bauer, W. Simeth, S. Mühlbauer, H. Berger, M. Garst, A. Rosch, and C. Pfleiderer, Observation of two independent skyrmion phases in a chiral magnetic material, Nat. Phys. 14, 936 (2018)

[39] F. Qian, L. J. Bannenberg, H. Wilhelm, G. Chaboussant, L. M. Debeer-Schmitt, M. P. Schmidt, A. Aqeel, T. T. M. Palstra, E. Brück, A. J. E. Lefering, C. Pappas, M. Mostovoy, and A. O. Leonov, New magnetic phase of the chiral skyrmion material $\mathrm{Cu}_{2} \mathrm{OSeO}_{3}$, Sci. Adv. 4, eaat7323 (2018).

[40] I. Stasinopoulos, S. Weichselbaumer, A. Bauer, J. Waizner, H. Berger, S. Maendl, M. Garst, C. Pfleiderer, and D. Grundler, Low spin wave damping in the insulating chiral magnet $\mathrm{Cu}_{2} \mathrm{OSeO}_{3}$, Appl. Phys. Lett. 111, 032408 (2017).

[41] S. L. Zhang, A. Bauer, D. M. Burn, P. Milde, E. Neuber, L. M Eng, H. Berger, C. Pfleiderer, G. van der Laan, and T. Hesjedal, Multidomain skyrmion lattices state in $\mathrm{Cu}_{2} \mathrm{OSeO}_{3}$, Nano Lett. 16, 3285 (2016).

[42] A. Vansteenkiste, J. Leliaert, M. Dvornik, M. Helsen, F. GarciaSanchez, and B. van Waeyenberge, The design and verification of MuMax3, AIP Adv. 4, 107133 (2014).

[43] O. Janson, I. Rousochatzakis, A. A. Tsirlin, M. Belesi, A. A. Leonov, U. K. Rößler, J. van den Brink, and H. Rosner, The quantum nature of skyrmions and half-skyrmions in $\mathrm{Cu}_{2} \mathrm{OSeO}_{3}$, Nat. Commun. 5, 5376 (2014).

[44] S. L. Zhang, G. van der Laan, J. Müller, L. Heinen, M. Garst, A. Bauer, H. Berger, C. Pfleiderer, and T. Hesjedal, Reciprocal space tomography of 3D skyrmion lattice order in a chiral magnet, Proc. Natl. Acad. Sci. USA 115, 6386 (2018).

[45] B. A. Kalinikos, and A. N. Slavin, Theory of dipole-exchange spin wave spectrum for ferromagnetic films with mixed exchange boundary conditions, J. Phys. C: Solid State Phys. 19 7013 (1986).

[46] D. D. Stancil and A. Prabhakar, Spin Waves: Theory and Applications (Springer, New York, 2009), Chap. 5, pp. 139-166.

[47] https://doi.org/10.5281/zenodo.5062921. 